\title{
Bronchial lumen is the safer route for an airway exchange catheter in double-lumen tube replacement: preventable complication in airway management for thoracic surgery
}

\author{
Hsiang-Ling Wu ${ }^{1}$, Ying-Hsuan Tai ${ }^{1}$, Ling-Fang Wei ${ }^{2}$, Hung-Wei Cheng ${ }^{1}$, Chiu-Ming Ho ${ }^{1}$ \\ ${ }^{1}$ Department of Anesthesiology, Taipei Veterans General Hospital and National Yang-Ming University, Taipei, Taiwan; ${ }^{2}$ Soochow University Library, \\ Soochow University, Taipei, Taiwan \\ Correspondence to: Chiu-Ming Ho, MD, PhD. Department of Anesthesiology, Taipei Veterans General Hospital, No. 201, Sec. 2, Shipai Rd., Beitou \\ District, Taipei City 11217, Taiwan. Email: cmho@vghtpe.gov.tw.
}

\begin{abstract}
There is no current consensus on which lumen an airway exchange catheter (AEC) should be passed through in double-lumen endotracheal tube (DLT) to exchange for a single-lumen endotracheal tube (SLT) after thoracic surgery. We report an unusual case to provide possible solution on this issue. A 71-year-old man with lung adenocarcinoma had an event of a broken exchange catheter used during a DLT replacement with a SLT, after a video-assisted thoracic surgery. The exchange catheter was impinged at the distal tracheal lumen and snapped during manipulation. All three segments of the catheter were retrieved without further airway compromises. Placement of airway tube exchanger into the tracheal lumen of doublelumen tube is a potential contributing factor of the unusual complication. We suggest an exchange catheter be inserted into the bronchial lumen in optimal depth with the adjunct of video laryngoscope, as the safe method for double-lumen tube exchange.
\end{abstract}

Keywords: Endotracheal tube exchanger; extubation; tracheal tube exchange

Submitted Mar 10, 2017. Accepted for publication Aug 14, 2017.

doi: $10.21037 /$ jtd.2017.08.163

View this article at: http://dx.doi.org/10.21037/jtd.2017.08.163

\section{Introduction}

Replacement of a double-lumen endotracheal tube (DLT) with a single-lumen endotracheal tube (SLT) is routinely performed for postoperative ventilator support in selected thoracic surgery patients. The technique can be challenging when attempted in patients with a difficult airway. An airway exchange catheter (AEC) facilitates exchanging from a DLT to a SLT, or vice versa (1-3). However, various AECassociated complications have been revealed, including laryngeal trauma (4), tracheobronchial trauma $(5,6)$, pneumothorax (4,7-9), lung laceration (10), esophageal perforation (11), and dislodgement of endotracheal tube (4). We present an unusual case in which an AEC was broken during exchange of a DLT to a SLT. This report adds to an insight regarding a novel complication associated with the use of AEC during the exchange process after thoracic surgery.

\section{Case presentation}

A 71-year-old man (height $157 \mathrm{~cm}$, weight $64 \mathrm{~kg}$; ASA class III) was well until left chest wall pain off and on with productive cough developed for two months; general weakness and poor appetite was also noted. His past history was unremarkable except for essential hypertension and type 2 diabetes mellitus. Physical examination on this admission was not remarkable. Chest $\mathrm{X}$-ray and computed tomography demonstrated a $4.3 \mathrm{~cm}$ soft-tissue density mass in left upper lobe of lung, in favor of tumor growth. Fiberbronchoscopy was performed under local anesthesia and specimens were collected; cytology and pathology revealed pulmonary adenocarcinoma. Therefore, a left upper lobectomy and radical lymph node dissection under video-assisted thoracoscopy was scheduled for the next day. The routine preoperative assessment and examinations were normal, except that the patient was anemic (hemoglobin, 

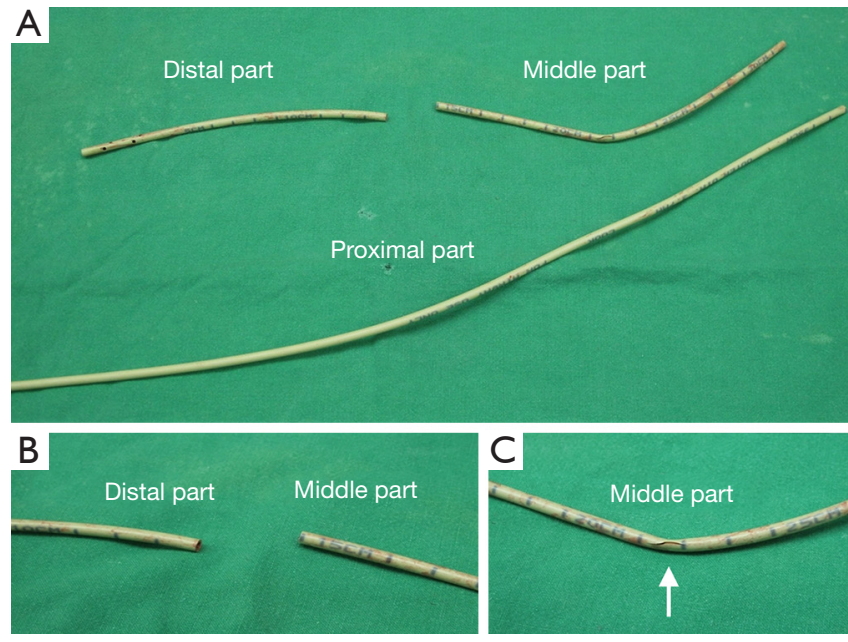

Figure 1 The broken airway exchange catheter. (A) Three segments of the broken airway exchange catheter; (B) the distal and middle parts were snapped at $15 \mathrm{~cm}$-mark; (C) a crack (arrow) was noted over the middle segment of the airway exchange catheter.

$11.8 \mathrm{~g} / \mathrm{dL}$ ). Preoperative airway assessment revealed limited mouth opening ( 2 fingers held vertically), with the prominent and sharp upper right maxillary canine, but full range of neck flexion and extension, and an appropriate thyromental distance.

On arrival at the operating theater, after standard monitors were applied, induction was achieved by total intravenous anesthesia with initial effect site concentration of $4.0 \mu \mathrm{g} / \mathrm{mL}$ of propofol and fentanyl $200 \mu \mathrm{g}$. After rocuronium $50 \mathrm{mg}$ for muscle relaxation, intubation of a 37 Fr, left-sided DLT (Mallinckrodt Endobronchial Tube, Covidien, Ireland) was attempted. At the first three attempts, the DLTs were all intubated into trachea with ruptured cuff torn by the teeth. The upper right maxillary canine was dislodged at the third time. At the fourth attempt, the DLT was successfully intubated and secured at $28 \mathrm{~cm}$ at the right angle of the mouth with adhesive tape. Flexible bronchoscopy was performed to confirm the DLT position at appropriate depth. Anesthesia was maintained with $60 \%$ oxygen/air mixture, continuous infusion of propofol, and thoracic epidural analgesia $(1.5 \%$ lidocaine $10 \mathrm{~mL}$ and fentanyl $50 \mu \mathrm{g}$ as loading dose; $0.25 \%$ bupivacaine $3.6-7.0 \mathrm{~mL} / \mathrm{h}$ as maintenance dose). The operation proceeded uneventfully.

At the end of the surgery, replacement of a DLT with a SLT was intended with the use of an AEC given concern of potentially difficult reintubation. We placed a welllubricated Cook AEC (11 Fr, 83 cm, Cook Critical Care,
USA) carefully through the tracheal lumen of DLT to the $25-\mathrm{cm}$ mark at the teeth. Severe resistance was met when we attempted to withdraw the DLT over the AEC, which stretched, snapped and was then pulled out of the trachea along with the DLT. At that time, the broken end of the AEC was noted (Figure 1A, the proximal part). Direct laryngoscopy revealed the distal part of AEC $(15 \mathrm{~cm}$-mark to distal end; Figure $1 A, B)$ left between vocal cords and the middle part (15 to $32 \mathrm{~cm}$-mark; Figure $1 A, C$ ) at the right retromolar trigone. We retrieved both segments with Magill forceps. Then, a fresh 7.5-mm SLT was repositioned subsequently under direct vision of laryngoscopy. There were no further adverse events, such as hypoxemia or tracheobronchial trauma. At postoperative intensive care unit, chest radiograph showed no new lesion, such as aspiration or foreign body. The patient was then weaned from mechanical ventilation soon after the surgery and uneventfully discharged one week later.

\section{Discussion}

Fracture of AEC during tube exchange after thoracic surgery is unusual but potentially lethal in airway management; it may result in airway obstruction with acute hypoxemia. A protruded broken end might also cause tracheobronchial trauma. In our patient, the distal AEC was caught and broken at the tracheal lumen outlet when the DLT was pulled. We believe that introduction of the AEC through the tracheal lumen of the DLT was the major contributing factor.

The DLT is a bifurcated tube with both a tracheal lumen and a bronchial lumen in order to selectively ventilate the left or right lung. Currently, there is no evidence-based consensus regarding either tracheal lumen or bronchial lumen for AEC insertion during replacement of a DLT with a SLT. An operator would much likely exposure the distal AEC when inserting the device into a tracheal lumen of DLT in usual practice (1), as it was in our patient (Figure 2A). We reason that it is potential for the exposed catheter tip to be bent and impinged in the trachea during this manipulation. Additionally, the distal orifice of tracheal lumen is gibbous-shaped with a $45^{\circ}$ bevel and smaller crosssectional area compared with bronchial lumen (Figure 2B), which might be associated with trap of AEC at the distal site. In contrast, placement of an AEC through the bronchial lumen in optimal depth maintains the nature curvature of catheter to prevent it from protruding pass the patient end of the DLT (Figure 2C), which may reduce the 

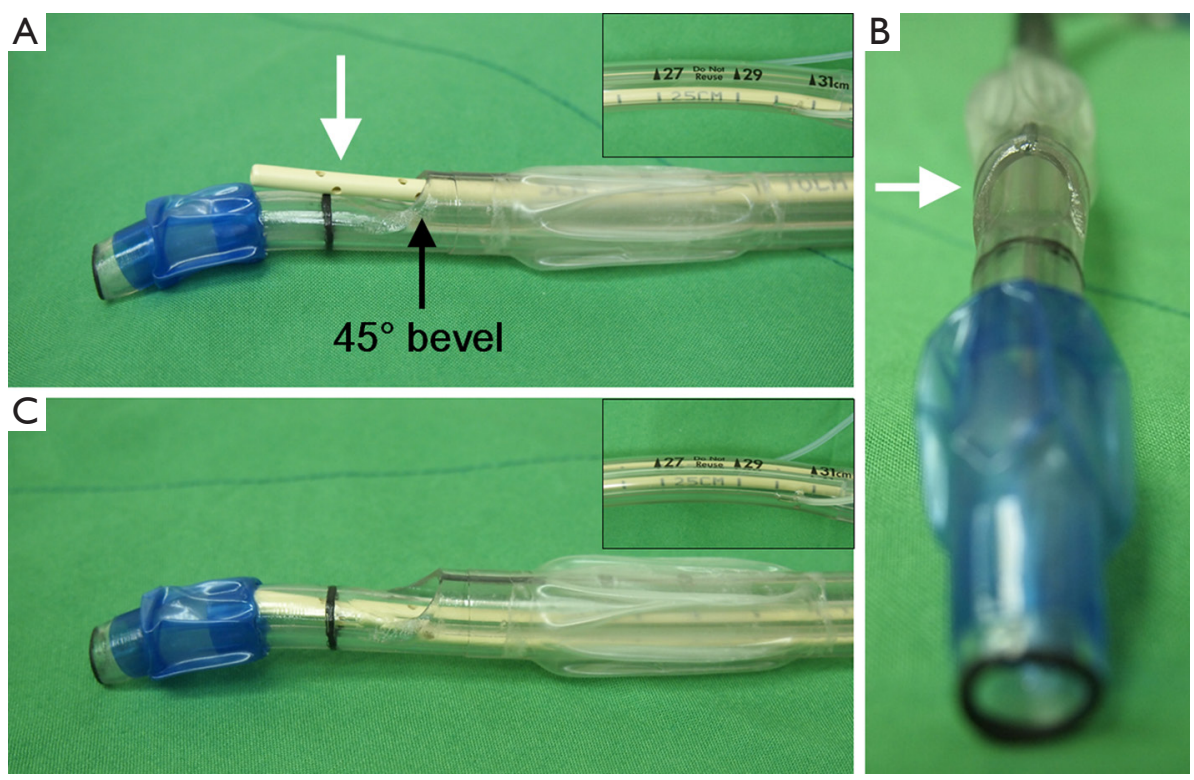

Figure 2 The distal airway exchange catheter is exposed when inserted into a tracheal lumen (arrow) (A) in contrast to a bronchial lumen (C) in the same depth (an $11 \mathrm{Fr}$ Cook AEC inserted to the $25-\mathrm{cm}$ mark into a $37 \mathrm{Fr}$, left-sided Mallinckrodt DLT maintained at $28 \mathrm{~cm}$ at the teeth as simulation); (B) the distal orifice (arrow) of tracheal lumen is gibbous-shaped with a $45^{\circ}$ bevel and smaller cross-sectional area compared with bronchial lumen.

risk of airway laceration or even perforation. Furthermore, the large distal orifice of bronchial lumen facilitates air escape and decreases gas flow if jet ventilation is delivered via an AEC (12), and this prevents potential barotraumas and even pneumothorax (13). Thus, the bronchial lumen of DLT may be suitable for the AEC passed during this replacement with a SLT, after thoracic surgery for postoperative ventilator support.

AECs can be classified into hollow and solid designs (14). Hollow catheters serve as a conduit to administer oxygen by jet ventilation and to intermittently measure partial pressure of end-tidal carbon dioxide from the trachea. However, hollow catheters withstand less tensile strength than solid ones when being stretched, which means vulnerable to fracture, as it was in the current patient. Although small-sized AECs can be more easily tolerated by conscious patients (15), it may be associated with easier kinks and fractures compared with large-sized ones. Thus, a large-sized and solid AEC might be a safer choice for a deeply-sedated patient during an exchange of a DLT with a SLT, if there is no need for jet ventilation or graphical measurement of carbon dioxide partial pressure.

The AEC allows exchange the DLT with a SLT in blind manner. However, previous works showed that adjunct laryngoscopy for this procedure may assist with not only continuous glottis viewing, but also clearing the pathway for AEC (16). In fact, adjunct video laryngoscopy may result in better glottis viewing and fewer airway complications $(16,17)$. If resistance of AEC is encountered during removing a DLT, as in this patient, performing adjunct video laryngoscopy to inspect the paraglottic structures during this procedure is necessary.

Based on the experience of our patient, to prevent distal impingement of AEC in DLT replacement, we make the following suggestions: (I) the AEC should be introduced through the bronchial lumen in optimal depth; (II) choose a large-sized and solid AEC for a deeply-sedated patient if there is no need for jet ventilation or capnographic monitoring; (III) keep continuous glottic viewing with a video laryngoscopy during withdrawing a DLT; (IV) airway gadgets need not be pulled against resistance, including AEC. If AEC fracture occurs, it is important to assure complete retrieval of AECs and sufficient ventilation.

In conclusion, we describe the successful management of fracture of an AEC, the potentially life-threatening complication, during replacement of a DLT to a SLT after thoracic surgery. We suggest an AEC be inserted into the bronchial lumen in optimal depth with the adjunct of video laryngoscopy as the safe method for exchange DLT for SLT. 


\section{Acknowledgements}

Funding: This study was support by the Research Fund (V106C-004) from Taipei Veterans General Hospital, Taipei, Taiwan.

\section{Footnote}

Conflicts of Interest: The authors have no conflicts of interest to declare.

Informed Consent: Written informed consent was obtained from the patient for publication of this manuscript and any accompanying images.

\section{References}

1. Hannallah M. Evaluation of tracheal tube exchangers for replacement of double-lumen endobronchial tubes. Anesthesiology 1992;77:609-10.

2. Cooper RM. The use of an endotracheal ventilation catheter in the management of difficult extubations. Can J Anaesth 1996;43:90-3.

3. Popat M, Mitchell V, Dravid R, et al. Difficult airway society guidelines for the management of tracheal extubation. Anaesthesia 2012;67:318-40.

4. McLean S, Lanam CR, Benedict W, et al. Airway exchange failure and complications with the use of the Cook Airway Exchange Catheter®: a single center cohort study of 1177 patients. Anesth Analg 2013;117:1325-27.

5. Seitz PA, Gravenstein N. Endobronchial rupture from endotracheal reintubation with an endotracheal tube guide. J Clin Anesth 1989;1:214-7.

6. Thomas V, Neustein SM. Tracheal laceration after the use of an airway exchange catheter for double-lumen tube placement. J Cardiothorac Vasc Anesth 2007;21:718-9.

7. Baraka AS. Tension pneumothorax complicating jet ventilation via a cook airway exchange catheter. Anesthesiology 1999;91:557-8.

8. Harris K, Chalhoub M, Maroun R, et al. Endotracheal tube exchangers: should we look for safer alternatives? Heart Lung 2012;41:67-9.

9. Rashid AM, Williams C, Noble J, et al. Pneumothorax, an underappreciated complication with an airway exchange catheter. J Thorac Dis 2012;4:659-62.

10. deLima LG, Bishop MJ. Lung laceration after tracheal extubation over a plastic tube changer. Anesth Analg 1991;73:350-1.

11. Fetterman D, Dubovoy A, Reay M. Unforeseen esophageal misplacement of airway exchange catheter leading to gastric perforation. Anesthesiology 2006;104:1111-2.

12. Dworkin R, Benumof JL, Benumof R, et al. The effective tracheal diameter that causes air trapping during jet ventilation. J Cardiothorac Anesth 1990;4:731-6.

13. Benumof JL. Airway exchange catheters: simple concept, potentially great danger. Anesthesiology 1999;91:342-4.

14. Cooper RM, Khan S. Extubation and reintubation of the difficult airway. In: Hagberg CA, editor. Benumof and Hagberg's airway management. Mosby; 2013, p. 1018-46.

15. Higgs A, Swampillai C, Dravid R, et al. Re-intubation over airway exchange catheters-mind the gap. Anaesthesia 2010;65:859-60.

16. Mort TC, Braffett BH. Conventional versus video laryngoscopy for tracheal tube exchange: glottic visualization, success rates, complications, and rescue alternatives in the high-risk difficult airway patient. Anesth Analg 2015;121:440-8.

17. Gamez R, Slinger P. A simulator study of tube exchange with three different designs of double-lumen tubes. Anesth Analg 2014;119:449-53.
Cite this article as: Wu HL, Tai YH, Wei LF, Cheng HW, Ho $\mathrm{CM}$. Bronchial lumen is the safer route for an airway exchange catheter in double-lumen tube replacement: preventable complication in airway management for thoracic surgery. J Thorac Dis 2017;9(10):E903-E906. doi: 10.21037/jtd.2017.08.163 